\title{
Role of tesmin expression in non-small cell lung cancer
}

\author{
JEDRZEJ GRZEGRZOLKA ${ }^{1}$, MATEUSZ OLBROMSKI ${ }^{1}$, AGNIESZKA GOMULKIEWICZ $^{1}$, \\ ALEKSANDRA PIOTROWSKA ${ }^{1}$, NATALIA GLATZEL-PLUCINSKA ${ }^{1}$, KATARZYNA RATAJCZAK ${ }^{1}$, \\ PATRYCJA SPUTA-GRZEGRZOLKA ${ }^{2}$, ADAM RZECHONEK ${ }^{3}$, BOZENA WERYNSKA ${ }^{4}$, \\ MARZENA PODHORSKA-OKOLOW ${ }^{5}$ and PIOTR DZIEGIEL ${ }^{1,6}$
}

\author{
Departments of ${ }^{1}$ Histology and Embryology, ${ }^{2}$ Anatomy, ${ }^{3}$ Thoracic Surgery, ${ }^{4}$ Pulmonology and Lung Cancer, and \\ ${ }^{5}$ Division of Ultrastructure Research, Wroclaw Medical University, 50-368 Wroclaw; \\ ${ }^{6}$ Department of Physiotherapy, Wroclaw University School of Physical Education, 51-612 Wroclaw, Poland
}

Received August 28, 2020; Accepted October 28, 2020

DOI: 10.3892/ol.2020.12309

\begin{abstract}
Non-small cell lung cancer (NSCLC) is the most commonly diagnosed cancer and the most frequent cause of cancer-associated mortality worldwide. Tesmin (MTL5) is a $60 \mathrm{kDa}$ protein which has cysteine rich motifs, characteristic of metallothioneins. Tesmin expression was first observed in germ cells during spermatogenesis. Increased tesmin expression in NSCLC has been described previously. Minichromosome maintenance proteins (MCMs) serve a critical role in replication and cell cycle progression, i.e. in NSCLC. The aim of the present study was to evaluate the localization and intensity of tesmin, MCM5 and MCM7 protein expression in NSCLC and their association with the clinicopathological data of patients. Archival paraffin blocks of 243 cases of NSCLC and 104 non-cancerous tissue samples from the surgical margin (control) were obtained from patients treated at the Clinic of Thoracic Surgery of Wroclaw Medical University (Wroclaw, Poland) between 2010 and 2016, and were used for tissue microarrays and immunohistochemical (IHC) experiments. Laser capture microdissection was used for the isolation of cancer cells from 36 frozen samples of NSCLC and 8 control samples, and subsequently, MTL5, MCM5 and $M C M 7$ mRNA expression was detected separately by reverse transcription-quantitative PCR. Positive cytoplasmic and nuclear tesmin, as well as nuclear MCM5 and MCM7 IHC expression were observed in $95.1,83.67,95.51$ and $100 \%$ of the NSCLC cases, respectively. MTL5, MCM5 and MCM7 mRNA expression was observed in $91.66 \%$ of the cancer cases for all genes. The statistical analysis revealed increased tesmin IHC expression in cancer cells compared with the control. A
\end{abstract}

Correspondence to: Mr. Jedrzej Grzegrzolka, Department of Histology and Embryology, Wroclaw Medical University, Chalubinskiego 6a, 50-368 Wroclaw, Poland

E-mail: jedrzej.grzegrzolka@umed.wroc.pl

Key words: non-small cell lung cancer, lung cancer, tesmin, minichromosome maintenance protein 5, minichromosome maintenance protein 7 , proliferation positive correlation was observed between the IHC expression of nuclear tesmin and MCM5 proteins $(\mathrm{r}=0.33 ; \mathrm{P}<0.0001)$ and nuclear tesmin and MCM7 proteins $(\mathrm{r}=0.315 ; \mathrm{P}<0.0001)$. In addition, a positive correlation between the mRNA expression levels of MTL5 and MCM5 ( $\mathrm{r}=0.421 ; \mathrm{P}<0.05)$, MTL5 and $M C M 7$ ( $\mathrm{r}=0.557 ; \mathrm{P}<0.01)$ was demonstrated. The survival analysis revealed that the presence of IHC cytoplasmic tesmin expression was a positive prognostic marker in NSCLC $(\mathrm{P}=0.0524)$. Furthermore, in vitro experiments performed on the NCI-H1703 cell line revealed that silencing of MTL5 mRNA and tesmin caused the downregulation of the expression levels of MCM5 and MCM7 and decreased the number of cells in the G2 phase. A positive association among tesmin, MCM5 and MCM7 could indicate a possible role of tesmin in the proliferation of NSCLC cancer cells.

\section{Introduction}

Lung cancer is currently one of the most common cancers and one of the most frequent causes of death worldwide (1). Non-small cell lung cancers (NSCLCs) constitute $~ 80 \%$ of all lung cancer cases. Adenocarcinoma, squamous cell carcinoma and large cell carcinoma are the three main histological subtypes of NSCLC, which account for 40, 25 and $10 \%$ of all the diagnosed cases of NSCLC, respectively (2). The course and prognosis of non-small cell lung cancers are associated with several factors, such as the histological type of cancer, the pTNM staging system and the presence of the EGFR gene mutation or the EML4-ALK fusion gene (3).

Tesmin (Testis-specific metallothionein-like protein, also known as metallothionein-like 5 protein, MTL5) is a $60 \mathrm{kDa}$ protein which has cysteine-rich motifs, characteristic of metallothioneins (MTs) (4). The human tesmin gene is located at 11q13.3 (5). The specific characteristics of this protein have been previously presented by us (6). Tesmin was first described in the mouse testicular tissue and rat testicular and ovarian tissues during the meiosis of male and female germ cells $(7,8)$. For that reason, a probable role of tesmin in the regulation of meiosis was suggested, and the hypothesis on the use of tesmin as a specific marker for germ cell differentiation was proposed. Moreover, the translocation from the cytoplasm to the nuclei 
of spermatocytes during the G2/M phase of the meiotic division may be indicative of the role of tesmin in the regulation of other genes involved in the process of spermatogenesis $(7,9)$. In addition, the nuclear expression of tesmin is suggested to be a response to heavy metal ions stress related to the presence of high concentrations of zinc and cadmium $(7,10)$. The expression of MTL5 mRNA was noted not only in murine ovaries and testes, but also in renal, brain, liver, and myocardial tissue. So far, the expression of tesmin in adult humans has been observed in prostate and gastric cancer $(11,12)$. An increased expression of tesmin was also observed in NSCLC, and it was positively correlated with the expression of the Ki-67 protein and associated with a poor prognosis (13).

The minichromosome maintenance protein complex (MCM) is a family of highly conservative, homogenous proteins (MCM2-MCM7) that play an active role in DNA synthesis (forming the prereplication-complex, which is a part of the replication forks) (14). The expression of MCM proteins is found in the early G1 phase, when the MCM2-MCM7 proteins interact with each other and form stable heterohexamers (15). MCM proteins expression increases during the whole cell cycle and decreases during the differentiation or G0 phase. They participate in the rearrangement of the chromatin structure, the maintenance of genomic stability, the cellular response associated with cell cycle checkpoints and the regulation of transcription $(16,17)$.

Many studies suggest that MCM proteins are expressed in cancer cells with a high proliferation activity. Consequently, they are considered as useful markers of proliferation in many cancers, including NSCLC $(18,19)$.

The aim of this study was to examine the expression of tesmin (MTL5), MCM5 and MCM7 in NSCLC cases, as well as their associations with the clinicopathological data of patients.

\section{Materials and methods}

Patients' characteristics. The clinical material consisted of 243 paraffin blocks from patients operated on for non-small cell lung cancer (NSCLC), including 92 cases of squamous cell lung carcinoma and 151 cases of lung adenocarcinoma. Additionally, 104 paraffin blocks from the surgical margin were used, which constituted the control for the analysed cases. In doubtful cases, immunohistochemical (IHC) anti-p63 (squamous cell lung carcinoma) and anti-TTF-1 (lung adenocarcinoma) reactions were performed in order to establish the final histopathological diagnosis. Moreover, the presence of necrosis in the neoplastic tumour was assessed by determining the percentage of the area of the histological preparation (stained with the HE method) covered by necrosis. After surgery, the median time that patients were followed for a 27 months (range: $0-81$ months, mean of $31.82 \pm 18.89$ ). Three patients did not report for a check-up after surgery. During the follow-up period, 95 patients died.

The tumour fragments and the tissue fragments from the surgical margin were frozen in liquid nitrogen, then the tumor cells and the normal lung tissue cells were isolated by laser microdissection and used to analyse MTL5, MCM5 and $M C M 7$ mRNA expressions. The median time that these cases were followed for was 24 months (range: 0-24 months, mean of 20.44 \pm 7.137 ). During the follow-up period, 18 patients died.
All patients were operated on in the years 2010-2016 at the Department of Thoracic Surgery of the Medical University in Wroclaw.

The specific clinicopathological data of the patients are presented in Table I.

Tissue microarrays (TMAs). The TMA method is a accepted method of archiving material in paraffin blocks with many advantages including economic aspects, stability of IHC reaction conditions and the time of evaluation of IHC results with relatively small restrictions (20). In our study 16 TMAs were prepared from 243 cases of NSCLC and 104 cases of control lung tissue from the surgical margin. Prior to performing TMA blocks the histological slides stained with haematoxylin and eosin were obtained from whole samples of NSCLC cases archived in the form of paraffin blocks (donor blocks). The slides were scanned using the Pannoramic Midi II histological scanner (3DHISTECH Ltd.). After that by using the Pannoramic Viewer Program (3DHISTECH Ltd.), the representative areas from the entire sections where selected. In addition, to increase the representativeness of each case, 3 representative cores with a size of $1.5 \mathrm{~mm}$ from the donor block were selected and then transferred to the TMA 'recipient' block using the TMA Grand Master (3DHISTECH Ltd.).

Immunohistochemistry. The paraffin blocks with NSCLC cases were cut into $4-\mu \mathrm{m}$ sections. The IHC reactions were performed using anti-tesmin rabbit polyclonal antibody (Novus Biologicals; cat. no. NBP2-13624) in a 1:400 dilution, anti-MCM5 (Santa Cruz Biotechnology, Inc.; sc-165994) in a 1:100 dilution, anti-MCM7 (Santa Cruz Biotechnology, Inc.; sc-9966) in a 1:50 dilution and anti-Ki-67 mouse monoclonal antibody Clone MIB-1 (Dako) ready-to-use. The IHC reactions were performed using the DAKO Autostainer Link 48 (Dako). The visualization of the reactions was carried out using EnVision $^{\mathrm{TM}}$ FLEX High pH (Link) reagents (Dako), according to the manufacturer's instructions. Positive IHC reaction for the tesmin antigen was assessed using the immunoreactive score (IRS) scale by Remmele and Stegner (21). This scale evaluates the percentage of positive cancer cells (A) and the staining intensity of the reaction (B). The final result is the product of these two values $(\mathrm{AxB})$. Following the antibody manufacturer instructions, before carrying out the IHC experiments, we performed reactions to the positive and negative controls.

Additionally, the nuclear expression intensity for the Ki-67, MCM5 and MCM7 proteins was determined using a scale that analyses the percentage of the number of cancer cells with positive nuclear expression of the antigens studied, according to the following scale: $0 \%, 0$ p.; 1-10\%, 1 p.; 11-25\%, 2 p.; 26-50\%, 3 p.; $51-100 \%, 4 \mathrm{p}$. All specimens were assessed using an OLYMPUS BX-41 light microscope (Olympus) by two independent pathologists. In cases with divergent scores the evaluation was reassessed and discussed until consensus was reached. The final result was the mean of the IHC expression scores of 3 TMA cores. Moreover, p63 and TTF-1 antigen expressions were used to confirm the histological type of the tumor (TTF-1(+) adenocarcinoma; p63(+) squamous cell carcinoma).

Cell line. Lung cancer cell line NCI-H1703 (lung squamous cell carcinoma) was obtained from the American Type Culture 
Table I. Patients and tumour characteristics.

\begin{tabular}{|c|c|c|}
\hline Parameter & IHC $(n=243)$ & RT-qPCR (n=36) \\
\hline $\begin{array}{l}\text { Mean age, } \\
\text { years (range) }\end{array}$ & $66.40 \pm 7.4(50-84)$ & $65.29 \pm 7.52(52-77)$ \\
\hline \multicolumn{3}{|l|}{ Sex, n (\%) } \\
\hline Male & $145(59.67)$ & $21(58.33)$ \\
\hline Female & $98(40.33)$ & $15(41.67)$ \\
\hline \multicolumn{3}{|c|}{ Tumour size, n (\%) } \\
\hline $\mathrm{T} 1$ & $76(31.28)$ & $8(22.22)$ \\
\hline $\mathrm{T} 2$ & $124(51.03)$ & $20(55.56)$ \\
\hline $\mathrm{T} 3$ & $24(9.88)$ & $5(13.89)$ \\
\hline $\mathrm{T} 4$ & $5(2.06)$ & $1(2.78)$ \\
\hline No data & $14(5.76)$ & $2(5.56)$ \\
\hline \multicolumn{3}{|c|}{ Lymph nodes, n (\%) } \\
\hline NO & $147(60.49)$ & $22(61.11)$ \\
\hline $\mathrm{N} 1, \mathrm{~N} 2, \mathrm{~N} 3$ & $82(33.74)$ & $12(33.33)$ \\
\hline No data & $14(5.76)$ & $2(5.56)$ \\
\hline \multicolumn{3}{|l|}{ Grade, n (\%) } \\
\hline G1 & $3(1.23)$ & $0(0.00)$ \\
\hline $\mathrm{G} 2$ & $150(61.73)$ & $25(69.44)$ \\
\hline G3 & $90(37.04)$ & $10(27.78)$ \\
\hline No data & $0(0.00)$ & $1(2.78)$ \\
\hline \multicolumn{3}{|l|}{ pTNM, n (\%) } \\
\hline I & $104(42.80)$ & $15(41.67)$ \\
\hline II & $76(31.28)$ & $15(41.67)$ \\
\hline III & $46(18.93)$ & $4(11.11)$ \\
\hline IV & $2(0.82)$ & $0(0.00)$ \\
\hline No data & $15(6.17)$ & $2(5.56)$ \\
\hline \multicolumn{3}{|l|}{ Stage, n (\%) } \\
\hline Early & $180(74.07)$ & $30(83.33)$ \\
\hline Advanced & $48(19.75)$ & $4(11.11)$ \\
\hline No data & $15(6.17)$ & $2(5.56)$ \\
\hline \multicolumn{3}{|c|}{ Histology, n (\%) } \\
\hline Adeno & $151(62.14)$ & $18(50.00)$ \\
\hline $\mathrm{SCC}$ & $92(37.86)$ & $18(50.00)$ \\
\hline \multicolumn{3}{|l|}{ p63,n (\%) } \\
\hline Positive & $111(45.68)$ & $20(55.56)$ \\
\hline Negative & 89 (36.63) & $11(30.56)$ \\
\hline No data & $43(17.70)$ & $5(13.89)$ \\
\hline \multicolumn{3}{|l|}{ TTF-1, n (\%) } \\
\hline Positive & $156(64.20)$ & $20(55.56)$ \\
\hline Negative & $44(18.11)$ & $11(30.56)$ \\
\hline No data & $43(17.70)$ & $5(13.89)$ \\
\hline \multicolumn{3}{|c|}{ Tesmin IHC, n (\%) } \\
\hline \multicolumn{3}{|l|}{ IRS } \\
\hline 0 & $12(4.94)$ & - \\
\hline $1-12$ & $231(95.06)$ & - \\
\hline \multicolumn{3}{|l|}{ Nuclear } \\
\hline 0 & $40(16.46)$ & - \\
\hline $1-4$ & $203(83.54)$ & - \\
\hline
\end{tabular}

IHC, immunohistochemistry; RT-qPCR, reverse transcriptionquantitative PCR; TTF-1, thyroid transcription factor 1; IRS, immunoreactive score; SCC, squamous cell carcinoma of the lung.
Collection. RPMI-1640 cell culture medium was used. The medium was additionally supplemented with L-glutamine to a final concentration of $2 \mathrm{mM}$, and with foetal bovine serum to a final concentration of $10 \%$. All of the cell culture media and reagents were provided by Sigma-Aldrich; Merck KGaA. In addition, we performed in vitro knock down experiments on the NCI-H1703 NSCLC cell line using Tesmin Silencer siRNAs s18519 and s18520 (Thermo Fisher Scientific, Inc.), receiving the silencing of the tesmin expression at a height of $\sim 55 \mathrm{kDa}$, as presented in our previous work (13). All experiments were performed in duplicate.

Cell cycle analyses by flow cytometry (FACS). The count of cultured cells (NCI-H1703) in the phases of the cell cycle was evaluated using the FACSCanto II flow cytometer (BD Biosciences) and FxCycle PI/RNase Staining Solution (Life Technologies; Thermo Fisher Scientific, Inc.), according to the manufacturer's instructions.

Laser capture microdissection (LCM). Frozen tissue samples of 36 NSCLC cases and 8 cases of non-malignant lung tissue (NMLT) (control) were used for RNA extraction. Tissue sections of $10-\mu \mathrm{m}$ thickness were prepared with use of a Leica CM1950 cryostat (Leica Microsystems) and placed on a polyethylene terephthalate membrane slide (MMI). The slides were fixed in $70 \%$ isopropyl alcohol and stained with HE by using the H\&E Staining Kit for LCM (MMI). LCM was performed using the MMI CellCut Plus System (MMI). The dissected neoplastic and normal cells were collected onto the adhesive lid of 500- $\mu 1$ tubes (MMI). Total RNA was isolated from the tissue samples by using the RNeasy Micro Kit (Qiagen) according to the manufacturer's instructions. The protocol included on-column DNase digestion to eliminate genomic DNA. First-strand cDNA was synthesized using the QuantiTect Reverse Transcription Kit (Qiagen), according to the manufacturer's instructions.

Reverse transcription-quantitative PCR (RT-qPCR). The mRNA expression of MTL5, MCM5 and MCM7 was studied on 36 cases of NSCLC and 8 control cases (18 cases of adenocarcinoma, 18 cases of squamous cell carcinoma and 8 cases of NMLT), as well as on cell cultures of NCI-H1703. Total RNA was extracted from the studied tissues and the cell line with the use of the RNeasy Mini Kit (Qiagen), according to the manufacturer's protocol. In order to eliminate genomic DNA contamination, on-column DNase digestion was performed using the RNase-Free DNase Set (Qiagen). The concentration and quality of the RNA samples were assessed by spectrophotometry using NanoDrop 1000 (Thermo Fisher Scientific, Inc.). First-strand cDNA was synthesized with the High Capacity cDNA Reverse Transcription Kit (Applied Biosystems; Thermo Fisher Scientific, Inc.). RT-qPCR was performed using the 7900HT Fast Real-Time PCR System and the TaqMan Gene Expression Master Mix (Applied Biosystems; Thermo Fisher Scientific, Inc.). $\beta$-actin $(A C T B)$ was used as an endogenous control. The following sets of primers and TaqMan probes were used in the studies: Hs01127481_m1 for MTL5,Hs01052148_m1 for MCM5, Hs00428518_m1 for MCM7 and Hs99999903_m1 for $A C T B$ (Applied Biosystems; Thermo Fisher Scientific, Inc.). The reactions were conducted in triplicates under the following 
conditions: polymerase activation at $50^{\circ} \mathrm{C}$ for $2 \mathrm{~min}$, denaturation at $94^{\circ} \mathrm{C}$ for $10 \mathrm{~min}$ followed by 40 cycles of denaturation at $94^{\circ} \mathrm{C}$ for $15 \mathrm{sec}$ and annealing with synthesis at $60^{\circ} \mathrm{C}$ for $1 \mathrm{~min}$. The relative expression of MTL5 mRNA was calculated using the $\Delta \Delta \mathrm{Cq}$ method (22).

Protein isolation, SDS-PAGE and western blotting. The Western blot technique (WB) was used to determine tesmin, MCM5 and MCM7 expression levels on the cell cultures of NCI-H1703. Whole protein lysates from the cell culture samples were obtained using the T-PER Tissue Protein Extraction Reagent (Thermo Fisher Scientific, Inc.) with the addition of the Halt ${ }^{\mathrm{TM}}$ Protease Inhibitor Cocktail (Thermo Fisher Scientific, Inc.) and 0.2 mM PMSF (Sigma-Aldrich; Merck KGaA). Protein concentrations were quantified using the Pierce BCA Protein Assay Kit (Thermo Fisher Scientific, Inc.) and the NanoDrop ${ }^{\mathrm{TM}} 1000$ (Thermo Fisher Scientific) spectrophotometer. Equal amounts of total protein $(30 \mu \mathrm{g})$ were mixed with Laemmli sample buffer and resolved on $10 \%$ acrylamide gel by SDS-PAGE. After electrophoresis, the samples were transferred to Immobilon-P polyvinylidene difluoride (PVDF) membranes (Merck KGaA) in the XCell SureLock $^{\mathrm{TM}}$ Mini-Cell Electrophoresis System (Thermo Fisher Scientific, Inc.). Next, the membranes were blocked in $4 \%$ bovine serum albumin solution (Merck KGaA) in TBST buffer (0.2 M Tris; $1.5 \mathrm{M} \mathrm{NaCl} ; 0.1 \%$ Tween-20). After blocking, the membranes were incubated overnight at $4^{\circ} \mathrm{C}$ with the primary rabbit anti-human tesmin polyclonal antibody (NBP2-13624; Novus Biologicals), diluted at 1:200, anti-MCM5 antibody (Santa Cruz Biotechnology, Inc.; sc-165994) diluted at 1:200 and anti-MCM7 (Santa Cruz Biotechnology, Inc.; sc-9966) antibody diluted at 1:200. Further, the membranes were incubated with secondary HRP-conjugated donkey anti-rabbit antibody (715-035-152; Jackson ImmunoResearch), diluted at 1:3,000 for $1 \mathrm{~h}$ at room temperature. Finally, the membranes were rinsed and treated with the Luminata Classico (Merck KGaA) chemiluminescent substrate. The reactions were visualized using the ChemiDoc Imaging System (Bio-Rad Laboratories). $\beta$-actin detected with primary rabbit anti-human $\beta$-actin antibody (4970; Cell Signaling Technology) diluted at 1:1,000 and secondary HRP-conjugated donkey anti-rabbit antibody (711-035-152; Jackson ImmunoResearch) diluted at 1:3,000 were used as an internal control to normalize the amount of tesmin. A densitometric analysis of the results obtained was performed with the use of the Image Lab software (Bio-Rad Laboratories).

Statistical analysis. The Kolmogorov-Smirnov test was used to evaluate the normality assumption of the examined groups. The Student's t-test, unpaired t-tests, Mann-Whitney, Kruskal-Wallis test with Dunn's multiple comparison test and MANOVA with Bonferroni post hoc tests were used to compare the differences in the expression of the examined markers in in vitro results and in all groups of patients and the clinicopathological data. Additionally, the Spearman's correlation test was used to analyse the existing correlations. The Kaplan-Meier method was used to construct survival curves. The Gehan-Breslow-Wilcoxon method and the univariate and multivariate Cox analyses of survival were performed to evaluate the analysis of survival. All statistical analyses
Table II. Correlations of IHC tesmin expression and MTL5 mRNA expression in cancer cells of non-small cell lung cancer ( $n=243$ for IHC analysis; $n=36$ for reverse transcriptionquantitative PCR analysis; Spearman correlation test).

\begin{tabular}{lcr}
\hline Markers & $\mathrm{r}$ & P-value \\
\hline Tesmin IRS vs. tesmin nuclear & -0.007 & 0.9086 \\
Tesmin IRS vs. Ki-67 & 0.015 & 0.8060 \\
Tesmin IRS vs. MCM5 & 0.049 & 0.4436 \\
Tesmin IRS vs. MCM7 & 0.034 & 0.5954 \\
Tesmin nuclear vs. Ki-67 & 0.239 & $<0.0010$ \\
Tesmin nuclear vs. MCM5 & 0.336 & $<0.0001$ \\
Tesmin nuclear vs. MCM7 & 0.315 & $<0.0001$ \\
Ki-67 vs. MCM5 & 0.634 & $<0.0001$ \\
Ki-67 vs. MCM7 & 0.603 & $<0.0001$ \\
MCM5 vs. MCM7 & 0.722 & $<0.0001$ \\
MTL5 vs. MCM5 & 0.421 & $<0.0500$ \\
MTL5 vs. MCM7 & 0.553 & $<0.0100$ \\
MCM5 vs. MCM7 & 0.861 & $<0.0001$ \\
\hline
\end{tabular}

IHC, immunohistochemistry; MTL5, tesmin; MCM, minichromosome maintenance protein; IRS, immunoreactive score.

were conducted using Prism 5.0 (GraphPad Software) and Statistica 13.3 (Tibco Software, Inc.). The results were considered statistically significant when $\mathrm{P}<0.05$.

\section{Results}

Cell line (in vitro tests). The analysis of the cell cycle by means of the assessment of the DNA content in the NCI-H1703 cells stained with propidium diodide and then analysed in the flow cytometer showed that in the NCI-H1703 cell line with a silenced MTL5 expression the percentage of cells in the G1/G0 phase increased significantly, while decreasing in the $\mathrm{G} 2 / \mathrm{M}$ phase (at $72 \mathrm{~h}$ of incubation) $(\mathrm{P}<0.0001$; MANOVA, Bonnferoni post hoc test; Fig. 1A).

Moreover, the statistical analysis of the results obtained by RT-qPCR showed a significantly lower expression of MTL5 mRNA in the line with the silenced expression of this gene compared to the control line, as well as no differences in the expression of MCM5 and MCM7 in the line with silenced MTL5 expression compared to the control line (Fig. 1B-D). In contrast, the expression of the tesmin, MCM5 and MCM7 proteins (analysed by means of optical density obtained by WB method) was lower in the line with silenced MTL5 expression than in the control line (Fig. 1E-H).

$R T-q P C R$. The analysis of the expression of the mRNA isolated by laser microdissection showed the expression of MTL5, MCM5 and MCM7 in $91.66 \%$ of cases. This expression was significantly higher in NSCLC compared to the control (Fig. 2A). There was no significant relationship between the expression of MTL5 and the histological type (Fig. 2B). Correlation analysis of mRNA expression: MTL5 and MCM5 as well as MTL5 and MCM7 showed statistically significant mean positive correlations (Table II). 
A

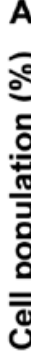

ฮั

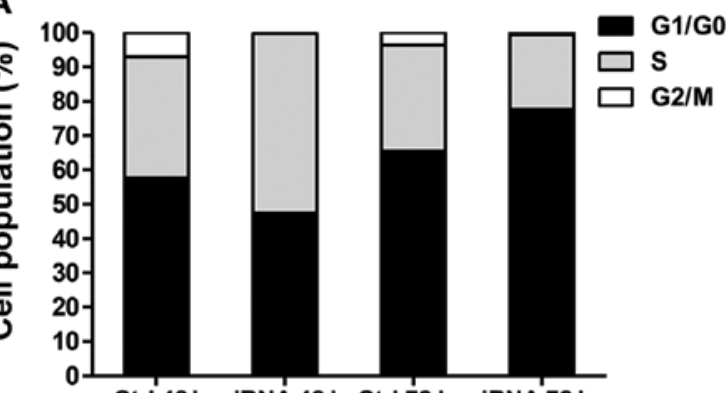

C

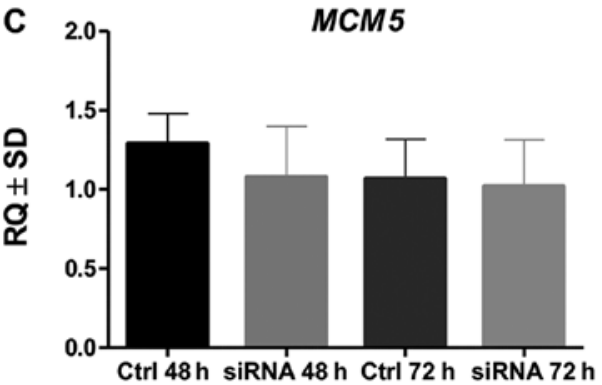

E
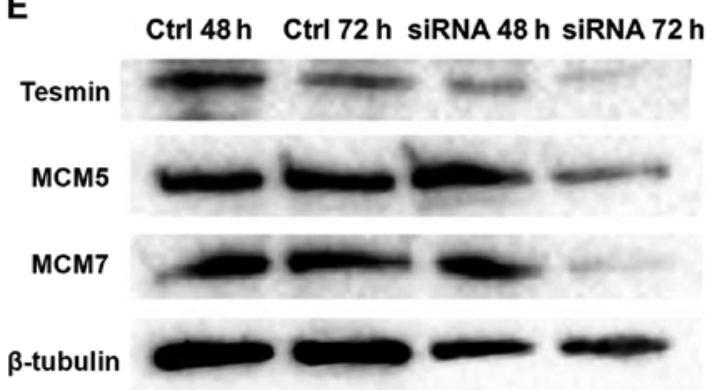

G

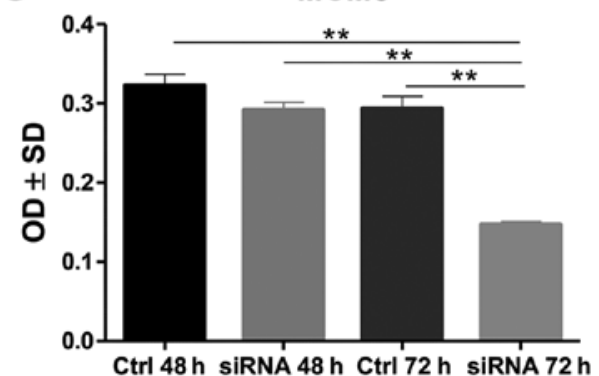

B

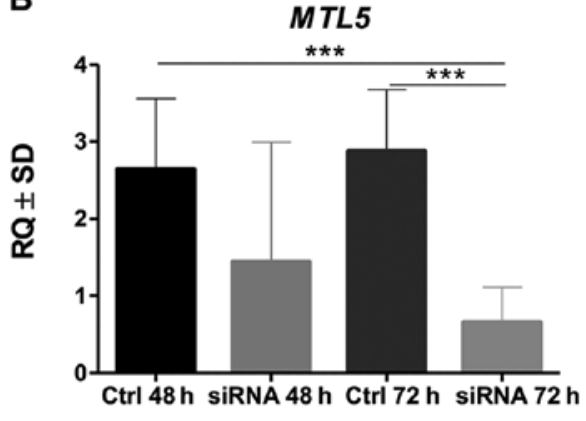

D

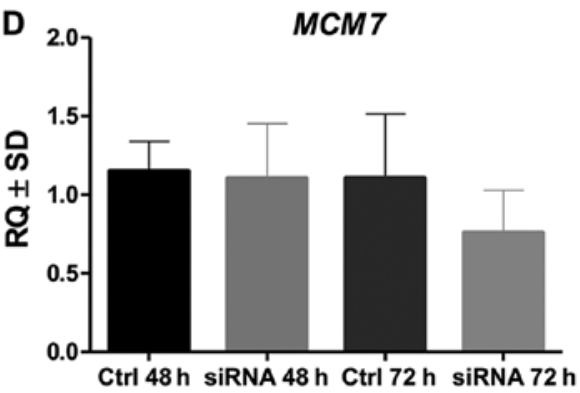

F

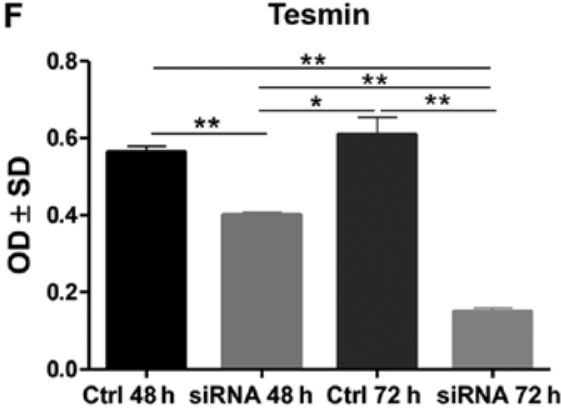

H

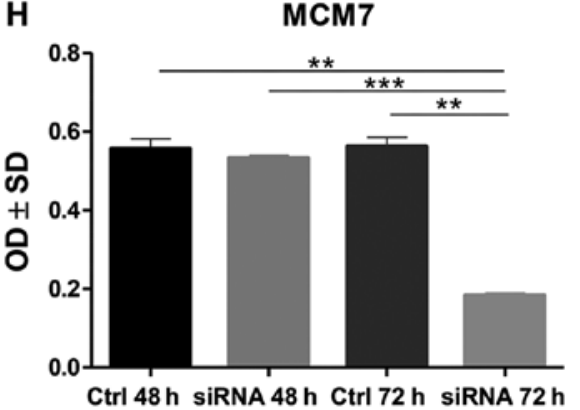

Figure 1. In vitro study of tesmin silencing in NCI-H1703 cell line. (A) Percentage distribution of NCI-H1703 cells in particular phases of the cell cycle in the control line (Ctrl) and with silenced expression of MTL5 mRNA (siRNA) after 48 and $72 \mathrm{~h}$ of incubation with MTL5 siRNA there was a reduction in the number of cells in G2/M phase. (B) Incubation with $M T L 5$ siRNA induced a significant decrease in $M T L 5$ mRNA expression at $72 \mathrm{~h}$ compared with controls at 48 and $72 \mathrm{~h}$. Incubation with $M T L 5$ siRNA did not significantly alter the mRNA expression of (C) MCM5 and (D) MCM7. (E) Western blot analysis of tesmin, MCM5 and MCM7 protein expression. The expression levels of tesmin as well as of MCM5 and MCM7 analysed by means of optical density obtained by the western blot analysis after $72 \mathrm{~h}$ of incubation with $M T L 5$ siRNA revealed a significantly lower expression of (F) tesmin protein, (G) MCM5 protein and (H) MCM7 protein. All experiments were performed in duplicate (Kruskal-Wallis test with Dunn's post hoc test; $\left.{ }^{*} \mathrm{P}<0.05 ;{ }^{* * *} \mathrm{P}<0.01 ;{ }^{* * * *} \mathrm{P}<0.001\right)$. Ctrl, control; MTL5, tesmin; siRNA, small interfering RNA; MCM, minichromosome maintenance protein; RQ, relative quantification; OD, optical density.

IHC. In the analysed NSCLC cases, positive cytoplasmic $(95.6 \%)$ and nuclear $(83.4 \%)$ IHC expression of tesmin were demonstrated (Fig. 3A-D). Both nuclear and cytoplasmic tesmin expressions were significantly higher in NSCLC cases compared to the control (Fig. 2C and D). Comparing the expression of tesmin in cases of squamous cell carcinoma showed a significantly higher nuclear expression of this protein in this histological type than in adenocarcinoma cases, while the cytoplasmic expression was significantly higher in cases of adenocarcinoma in comparison to squamous cell carcinoma cases (Fig. 2E and F). An analysis of the comparison of the expression of tesmin with the clinicopathological data of the NSCLC cases showed that nuclear tesmin expression was significantly lower in pT1 cases compared to pT2-T4 cases in both the whole NSCLC group and the squamous cell carcinoma group, but not in the adenocarcinoma group (respectively: $1.85 \pm 1.53$ vs. $2.37 \pm 1.48, \mathrm{P}<0.05 ; 1.49 \pm 1.45$ vs. $2.98 \pm 1.25$, $\mathrm{P}<0.0001 ; 1.85 \pm 1.53$ vs. $1.84 \pm 1.46, \mathrm{P}>0.05)$. Moreover, a mean 

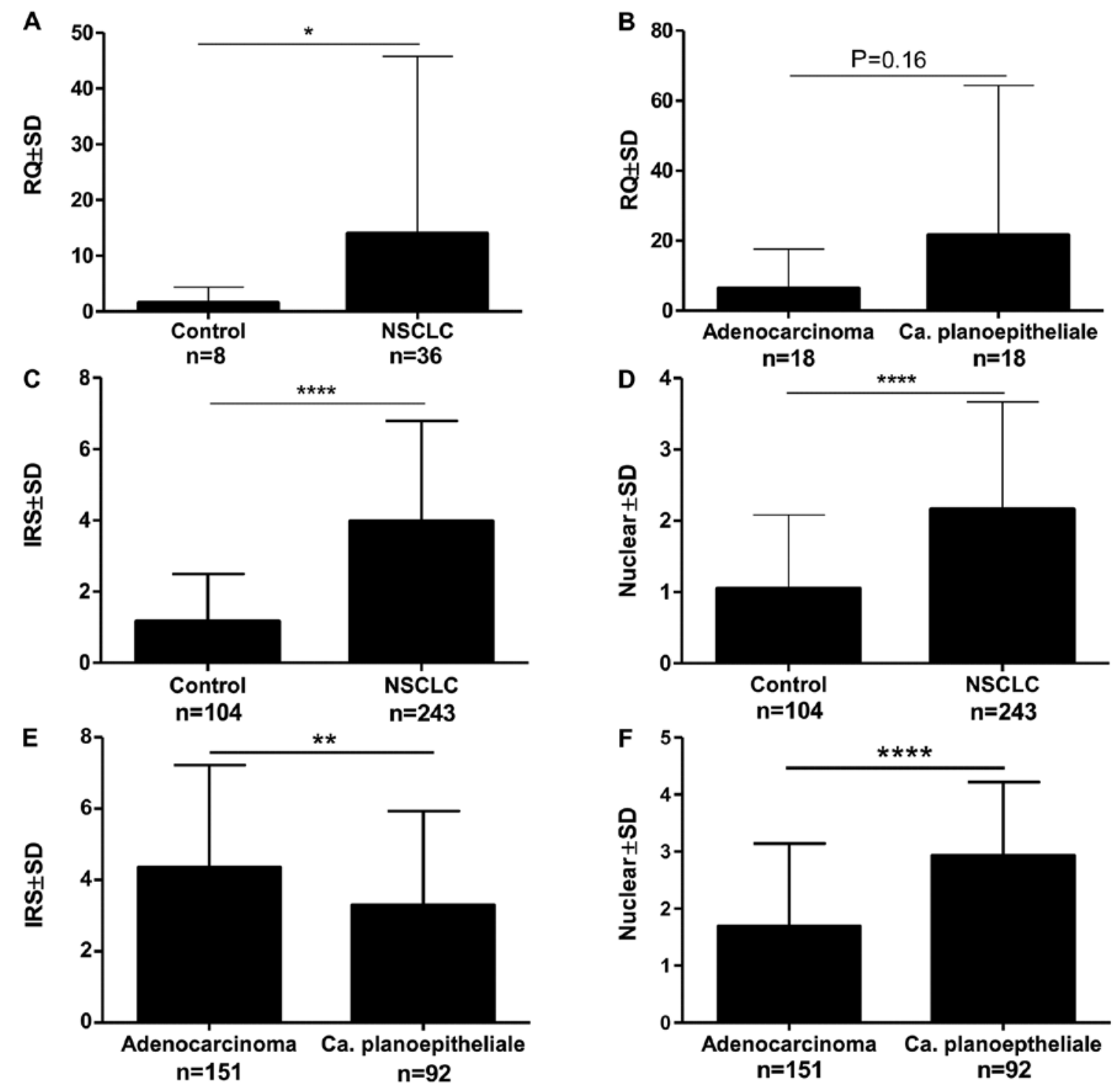

Figure 2. Analysis of the expression levels of MTL5 mRNA isolated by laser microdissection revealed (A) a significantly higher level of MTL5 mRNA expression in NSCLC cancer cells compared with the control, while (B) no significant differences were found in particular NSCLC subtypes. The statistical analysis of the immunohistochemistry expression of tesmin revealed that both (C) cytoplasmic and (D) nuclear expression levels were significantly higher in NSCLC cells compared with the control. (E) Cytoplasmic tesmin expression was higher in adenocarcinoma, while (F) nuclear expression was higher in squamous cell carcinoma (Mann-Whitney test; $\left.{ }^{*} \mathrm{P}<0.05 ;{ }^{* *} \mathrm{P}<0.01 ;{ }^{* * * * *} \mathrm{P}<0.0001\right)$. MTL5, tesmin; NSCLC, non-small cell lung cancer; RQ, relative quantification; IRS, immunoreactive score' NSCLC, Non-small cell lung cancer.

positive correlation between the tesmin nuclear expression and the percentage of the area of the preparation covered by necrosis was demonstrated $(\mathrm{r}=0.299, \mathrm{P}<0.0001)$, which was not observed for the cytoplasmic expression. The nuclear expression of tesmin in the NSCLC group correlated positively with the expression of the marker p63 $(r=0.464, \mathrm{P}<0.0001)$, and negatively with the expression of the marker TTF-1 ( $\mathrm{r}=-0.382$, $\mathrm{P}<0.0001)$. On the other hand, the cytoplasmic expression of tesmin in the NSCLC group correlated with the expression of the marker TTF-1 ( $\mathrm{r}=0.244, \mathrm{P}<0.001)$, while showing no correlation with the expression of the marker p63 ( $\mathrm{r}=-0.178, \mathrm{P}<0.05)$.

Additionally, no correlation was found between the expression of tesmin (both cytoplasmic and nuclear) and other clinicopathological data such as patient's age, sex, tumour size, pN, pM, clinical stage or tobacco smoking status (Fig. S1).

The analysis of the correlation of the intensity of the expression of tesmin (IHC) and the proliferation markers showed that the nuclear (but not the cytoplasmic) expression of tesmin correlated moderately positively with the nuclear expression of MCM5, MCM7 and Ki-67 (Fig. 3E-H; Table II).

Survival analysis. The analysis of the survival of the NSCLC cases showed that patients without confirmed (by the IHC method) cytoplasmic expression of tesmin in the tumour cells lived longer that patients with confirmed tesmin expression $(\mathrm{P}=0.052$, Gehan-Breslow-Wilcoxon test; Fig. 4A). This relationship was not demonstrated for the nuclear expression of tesmin (IHC). Similarly, patients with low MTL5 mRNA expression lived significantly longer than patients with high MTL5 mRNA expression $(\mathrm{P}<0.05$, Gehan-Breslow-Wilcoxon test; Fig. 4B). Moreover, univariate and multivariate Cox survival analyses showed that in NSCLC cases, only $\mathrm{pT}$ and $\mathrm{pN}$ are independent prognostic factors (Tables III-V). 

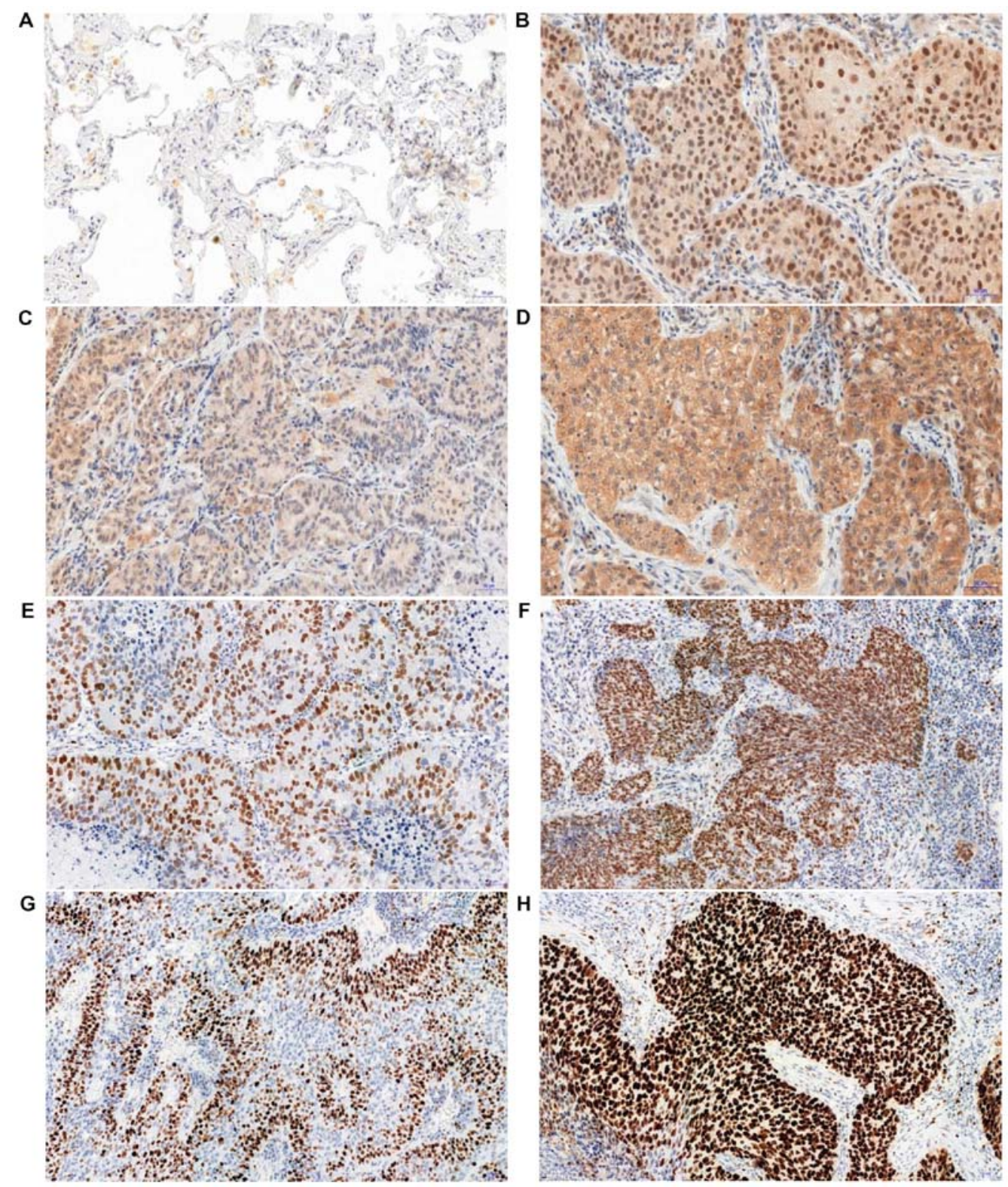

Figure 3. Immunohistochemical analysis of tesmin expression in controls and non-small cell lung cancer. (A) Low expression levels of tesmin in non-malignant lung tissue (mainly lung macrophages). (B) Nuclear-cytoplasmic expression of tesmin in non-small cell lung cancer cells. (C) Low and (D) high cytoplasmic expression of tesmin. (E) Low and (F) high MCM5 nuclear expression. (G) Low and (H) high MCM7 nuclear expression. Magnification, x200. MCM, minichromosome maintenance protein.
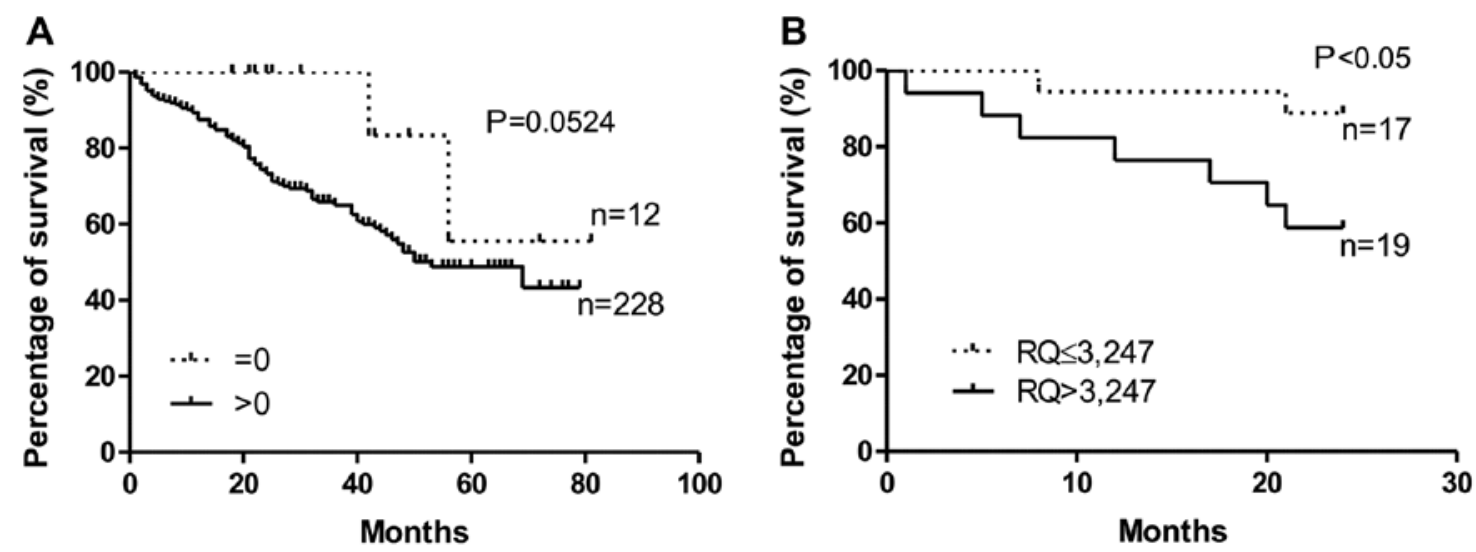

Figure 4. Kaplan-Meier survival curves and survival analysis of patients with high and low MTL5 and tesmin expression. (A) Elevated cytoplasmic expression levels of tesmin in NSCLC cells are an unfavourable prognostic factor. (B) Similarly, patients with an elevated tesmin mRNA expression (cut-off, median) in NSCLC cells had significantly shorter survival (Gehan-Breslow-Wilcoxon test). NSCLC, non-small cell lung cancer; RQ, relative quantification. 
Table III. Univariate and multivariate Cox analyses of overall survival of patients with non-small cell lung cancer.

\begin{tabular}{|c|c|c|c|c|c|c|c|c|}
\hline \multirow[b]{2}{*}{ Characteristics } & \multicolumn{4}{|c|}{ Univariate Cox analysis of survival } & \multicolumn{4}{|c|}{ Multivariate Cox analysis of survival } \\
\hline & P-value & $\begin{array}{l}\text { Hazard } \\
\text { ratio }\end{array}$ & $\begin{array}{c}95 \% \mathrm{CI} \\
\text { lower }\end{array}$ & $\begin{array}{c}95 \% \text { CI } \\
\text { upper }\end{array}$ & P-value & $\begin{array}{c}\text { Hazard } \\
\text { ratio }\end{array}$ & $\begin{array}{c}95 \% \mathrm{CI} \\
\text { lower }\end{array}$ & $\begin{array}{c}95 \% \text { CI } \\
\text { upper }\end{array}$ \\
\hline p63 (negative vs. positive) & 0.7645 & 1.0680 & 0.6945 & 1.6424 & & & & \\
\hline TTF-1 (negative vs. positive) & 0.8613 & 1.0484 & 0.6168 & 1.7822 & & & & \\
\hline Histological type (adeno vs. SCC) & 0.1486 & 0.7301 & 0.4765 & 1.1187 & & & & \\
\hline Clinical stage (I-II vs. III-IV) & 0.0003 & 1.5542 & 1.2215 & 1.9775 & 0.6312 & 1.1109 & 0.7231 & 1.7064 \\
\hline pT (pT1-2 vs. pT3-4) & 0.0024 & 1.5170 & 1.1593 & 1.9852 & 0.0061 & 1.7928 & 1.1809 & 2.7216 \\
\hline pN (pN0 vs. pN1-3) & 0.0023 & 1.9072 & 1.2604 & 2.8861 & 0.0068 & 1.4608 & 1.1105 & 1.9216 \\
\hline Necrosis (\%) (continuous) & 0.3824 & 0.9458 & 0.8347 & 1.0718 & & & & \\
\hline Histological grade (G1 vs. G2-3) & 0.1886 & 1.3095 & 0.8761 & 1.9572 & & & & \\
\hline Tesmin IRS (0 vs. 1-12) & 0.1184 & 3.0555 & 0.7521 & 12.4124 & & & & \\
\hline Tesmin nuclear (0 vs. $1-4)$ & 0.3831 & 1.2978 & 0.7225 & 2.3310 & & & & \\
\hline MCM-5 (0-2 vs. $3-4)$ & 0.4159 & 0.8169 & 0.5019 & 1.3297 & & & & \\
\hline MCM-7 (0-2 vs. $3-4)$ & 0.5114 & 1.3192 & 0.5770 & 3.0160 & & & & \\
\hline Ki-67 (0-2 vs. 3-4) & 0.8906 & 0.9641 & 0.5730 & 1.6224 & & & & \\
\hline
\end{tabular}

IRS, immunoreactive score; MCM, minichromosome maintenance protein; TTF-1, thyroid transcription factor 1; SCC, squamous cell carcinoma of the lung.

Table IV. Univariate and multivariate Cox analyses of overall survival of patients with lung adenocarcinoma.

\begin{tabular}{|c|c|c|c|c|c|c|c|c|}
\hline \multirow[b]{2}{*}{ Characteristics } & \multicolumn{4}{|c|}{ Univariate Cox analysis of survival } & \multicolumn{4}{|c|}{ Multivariate Cox analysis of survival } \\
\hline & P-value & $\begin{array}{c}\text { Hazard } \\
\text { ratio }\end{array}$ & $\begin{array}{c}95 \% \mathrm{CI} \\
\text { lower }\end{array}$ & $\begin{array}{c}95 \% \text { CI } \\
\text { upper }\end{array}$ & P-value & $\begin{array}{l}\text { Hazard } \\
\text { ratio }\end{array}$ & $\begin{array}{c}95 \% \mathrm{CI} \\
\text { lower }\end{array}$ & $\begin{array}{c}95 \% \text { CI } \\
\text { upper }\end{array}$ \\
\hline p63 (negative vs. positive) & 0.0508 & 1.7264 & 0.9982 & 2.9859 & 0.4915 & 1.1647 & 0.7543 & 1.7984 \\
\hline TTF-1 (negative vs. positive) & 0.3777 & 0.5910 & 0.1837 & 1.9012 & & & & \\
\hline Clinical stage (I-II vs. III-IV) & 0.0056 & 1.4877 & 1.1231 & 1.9707 & 0.5683 & 1.1346 & 0.7352 & 1.7510 \\
\hline pT (pT1-2 vs. pT3-4) & 0.0277 & 1.4084 & 1.0383 & 1.9104 & 0.0068 & 1.4608 & 1.1105 & 1.9216 \\
\hline pN (pN0 vs. pN1-3) & 0.0036 & 2.1450 & 1.2833 & 3.5852 & 0.0061 & 1.7928 & 1.1809 & 2.7216 \\
\hline Necrosis, \% (continuous) & 0.9781 & 0.9973 & 0.8222 & 1.2097 & & & & \\
\hline Histological grade (G1 vs. G2-3) & 0.1548 & 1.4197 & 0.8761 & 2.3007 & & & & \\
\hline Tesmin IRS (0 vs. 1-12) & 0.5856 & 0.7525 & 0.2707 & 2.0914 & & & & \\
\hline Tesmin nuclear (0 vs. 1-4) & 0.2839 & 1.4119 & 0.7514 & 2.6530 & & & & \\
\hline MCM-5 (0-2 vs. 3-4) & 0.6605 & 0.8876 & 0.5213 & 1.5112 & & & & \\
\hline MCM-7 (0-2 vs. 3-4) & 0.3379 & 1.5103 & 0.6499 & 3.5102 & & & & \\
\hline Ki-67 (0-2 vs. 3-4) & 0.9732 & 1.0101 & 0.5603 & 1.8212 & & & & \\
\hline
\end{tabular}

IRS, immunoreactive score; MCM, minichromosome maintenance protein; TTF-1, thyroid transcription factor 1.

\section{Discussion}

In the presented study, we have demonstrated for the first time a relationship between an increased nuclear expression of tesmin and a higher expression of MCM5 and MCM7 at the protein and mRNA levels in NSCLC. A positive correlation between the expression of tesmin and Ki-67 in NSCLC, which we have as well demonstrated in this study, was also previously observed by us (13). This indirectly indicates a relationship between the expression of tesmin and the proliferation process of NSCLC cells. The relationship of tesmin with the process of cellular proliferation has already been described on many occasions in the case of cells undergoing meiotic division (4,6-9). A higher expression of this protein and its translocation from the cytoplasm to the cell nucleus were observed, suggesting its participation in the organization of chromatin in chromosomes (6). Furthermore, this phenomenon was intensified by the presence of zinc and cadmium ions (6-9). 
Table V. Univariate Cox analysis of overall survival of patients with squamous cell carcinoma of the lung.

Univariate Cox analysis of survival

\begin{tabular}{lcccc}
\cline { 2 - 4 } Characteristics & P-value & Hazard ratio & 95\% CI lower & 95\% CI upper \\
\hline TTF-1 (negative vs. positive) & 0.7255 & 0.8774 & 0.4227 & 1.8213 \\
Clinical stage (I-II vs. III-IV) & 0.0614 & 1.6079 & 0.9775 & 2.6447 \\
pT (pT1-2 vs. pT3-4) & 0.0206 & 1.9206 & 1.1052 & 3.3377 \\
pN (pN0 vs. pN1-3) & 0.4125 & 1.3751 & 0.6420 & 2.9452 \\
Necrosis, \% (continuous) & 0.6559 & 0.9575 & 0.7908 & 1.1592 \\
Histological grade (G1 vs. G2-3) & 0.3497 & 0.5671 & 0.1727 & 1.8619 \\
Tesmin IRS (0 vs. 1-12) & 0.4507 & 1.7353 & 0.4143 & 7.2686 \\
Tesmin nuclear (0 vs. 1-4) & 0.5495 & 1.8384 & 0.2503 & 13.5045 \\
MCM-5 (0-2 vs. 3-4) & 0.9938 & 0.9920 & 0.1321 & 1.4486 \\
MCM-7 (0-3 vs. 4) & 0.3132 & 0.8703 & 0.5433 & 1.5824 \\
Ki-67 (0-2 vs. 3-4) & 0.4511 & 0.7507 & 0.3562 & \\
\hline
\end{tabular}

IRS, immunoreactive score; MCM, minichromosome maintenance protein; TTF-1, thyroid transcription factor 1.

The role of tesmin in the process of proliferation of NSCLC cells is additionally confirmed by an in vitro experiment in which the silencing of the expression of tesmin with siRNA causes a reduction in the percentage of cells in the $\mathrm{G} 2$ phase of the cell cycle and a reduction of the expression of MCM5 and MCM7, which are confirmed proliferation markers also in NSCLC $(16,17)$.

Despite this, the role of tesmin in cancer is not fully understood. An increased expression of this protein in NSCLC cells compared to the control tissue has already been described by us (13). However, contrary to the previous study, this time we were also able to show for the first time a difference in the localization of tesmin expression depending on the histological subtype of NSCLC. In cases of lung adenocarcinoma, the cytoplasmic expression of tesmin was higher than in cases of squamous cell carcinoma. In contrast, in cases of squamous cell carcinoma, the nuclear expression of tesmin was higher than in the adenocarcinomas. Similarly, the nuclear expression of MCM5 and MCM7 was also significantly higher in squamous cell carcinoma than in adenocarcinoma (data not shown in the study). This confirms that NSCLCs are a heterogeneous group of neoplasms, and squamous cell carcinomas and adenocarcinomas may have different regulatory mechanisms, something that has already been emphasized on numerous occasions (23-26). This may also indirectly indicate the participation of tesmin, MCM5 and MCM7 in the same mechanisms that influence carcinogenesis and progression in NSCLC, resulting, among others, in a higher proliferative potential of the cells for this type of tumour. A stronger nuclear expression of tesmin in squamous cell carcinoma and the alleged biological role of tesmin in the cell nucleus may suggest that tesmin plays a more important role in this subtype of NSCLC. This is confirmed by the fact that a different profile of genes and a different expression of markers in adenocarcinoma and squamous cell lung carcinoma have already been indicated numerous times (26).

The positive correlations between the expression of tesmin (MLT5) and MCM5 and MCM7 at the level of the protein (IHC) and the mRNA isolated from tumour cells by using the laser microdissection method may indicate the mutual regulation of these proteins. Sanada et al (27) suggested that the miR-143 molecule is involved in the regulation of the expression of MTL5 and another member of the MCM-MCM4 protein family, among others. Taking into account the already known function of the proteins from the MCM family in DNA replication, perhaps tesmin is also involved in this process, all the more so because there are reports that suggest a possible role of tesmin in the organization of chromatin during meiotic divisions (4). It is possible that tesmin has a similar function in carcinogenesis and mitotic divisions of NSCLC cells.

Due to the fact that tesmin is indicated as a co-activator that differentiates the activity of the ligands aldosterone and deoxycorticosterone for the mineralocorticoid receptor (this receptor is a ligand-dependent transcription factor), it can be assumed that tesmin, as a co-activator, may regulate the expression of other genes, e.g. those related to the proliferation process (28). However, this hypothesis would require further, more detailed research.

Moreover, as in our previous study, we were also able to demonstrate that an increased expression of tesmin is a negative prognostic factor in NSCLC, both at the mRNA and the IHC levels (13). A similar role of the expression of MTL5 was indicated by Sanada et al (27). This proves that tesmin may have a significant effect on the progression of NSCLC.

One of the limitation of the study was lack of clinical data in the form of presence of the EGFR gene mutation. This is due the fact that the mutation testing of NSCLC in Poland is not routinely performed in cases of I and II stage of non-small cell lung cancer. The cases in these stages that are mostly treated surgically and constitute the dominant group of cases in the presented study. Unfortunately, we also do not have such clinical data for a much smaller group of patients in stage III and IV. Because the main cause of the EGFR gene mutation is smoking we compared the expression of tesmin in smokers and non-smokers but we did not reveal any significant differences in the expression of tesmin in these groups. 
The present study was carried out using the NCI-H1703 squamous cell carcinoma because the analysis of MTL5 mRNA expression isolated by laser capture microdissection from NSCLC cases shown that the MTL5 expression was higher in squamous cell carcinoma cases than in adenocarcinoma cases. The results of IHC analysis also shown that the nuclear expression of tesmin was significantly higher in squamous cell carcinoma cases than in adenocarcinoma cases. Moreover, it seems that the biological activity of the tesmin protein is dominant in the cell nucleus $(7,28,29)$. In addition, on the basis of previous research in which we showed the highest nuclear expression of tesmin in the NCI-H1703 cell line we assumed that this cell line would be most suitable for our research (13). However using only one cell line could be also limitation of our study

In conclusion, the role of tesmin in NSCLC seems to be related to the cell proliferation process of this tumour, and a worse prognosis of NSCLC patients with a higher expression of it may indicate the role of this protein in the progression of NSCLC.

\section{Acknowledgements}

Not applicable.

\section{Funding}

The present study was supported by the National Science Centre, Poland, under the programme 'Preludium 12' project no. $2016 / 23 / \mathrm{N} / \mathrm{NZ} 5 / 02570$.

\section{Availability of data and materials}

The datasets used and/or analyzed during the current study are available from the corresponding author on reasonable request.

\section{Authors' contributions}

JG designed the study, evaluated the IHC reaction, prepared the database, performed the statistical analysis, analysed the results and created a draft of the manuscript. PSG evaluated the IHC reaction and prepared the database. AG performed microdissection, isolation of mRNA and quantitative PCR analysis. MO, KR and NGP prepared all in vitro experiments and analysed the results. AP prepared the IHC analysis and analysed the results. MPO, BW and AR obtained in vivo material from patients, collected clinical data of patients, prepared the database and analysed the results. MPO and PD reviewed and edited the manuscript. PD supervised methodical all experiments, analysed the results, prepared, reviewed and edited manuscript. All authors agree to be accountable for all aspects of the research in ensuring that the accuracy or integrity of any part of the work are appropriately investigated and resolved. All authors read and approved the final manuscript.

\section{Ethics approval and consent to participate}

The experiment was performed in accordance with the ethical standards and following approval of the Ethics Committee of
Wroclaw Medical University (decision no. KB 455/2009 and KB 40/2017) and all patients provided a written statement of informed consent for the use of the material samples for scientific research.

\section{Patient consent for publication}

Not applicable.

\section{Competing interests}

The authors declare that they have no competing interests.

\section{References}

1. Brambilla E and Travis WD: Lung cancer. In: Word Cancer Report 2014. Stewart BW and Wild CP (eds). World Health Organization, Lyon, pp350-361, 2014.

2. EttingerDS, Akerley W,Bepler G,Blum MG,Chang A, Cheney RT, Chirieac LR, D'Amico TA, Demmy TL, Ganti AK, et al; NCCN non-small cell lung cancer panel members: Non-small cell lung cancer. J Natl Compr Canc Netw 8: 740-801, 2010.

3. Camidge DR, Dziadziuszko R, Peters S, Mok T, Noe J, Nowicka M, Gadgeel SM, Cheema P, Pavlakis N, de Marinis F, et al: Updated efficacy and safety data and impact of the EML4-ALK fusion variant on the efficacy of alectinib in untreated ALK-positive advanced non-small cell lung cancer in the global phase III ALEX study. J Thorac Oncol 14: 1233-1243, 2019.

4. Sutou S, Miwa K, Matsuura T, Kawasaki Y, Ohinata Y and Mitsui Y: Native tesmin is a 60-kilodalton protein that undergoes dynamic changes in its localization during spermatogenesis in mice. Biol Reprod 68: 1861-1869, 2003.

5. HUGO Gene Nomenclature Committee: http://www.genenames. org. Accessed May 30, 2020.

6. Grzegrzółka J, Podhorska-Okołów M, Krawczuk Z and Dzięgiel P: The role of tesmin in the physiology and pathogenesis of human diseases. Postepy Hig Med Dosw 73: 762-767, 2019.

7. Matsuura T, Kawasaki Y, Miwa K, Sutou S, Ohinata Y, Yoshida F and Mitsui Y: Germ cell-specific nucleocytoplasmic shuttling protein, tesmin, responsive to heavy metal stress in mouse testes. J Inorg Biochem 88: 183-191, 2002.

8. Olesen C, Møller M and Byskov AG: Tesmin transcription is regulated differently during male and female meiosis. Mol Reprod Dev 67: 116-126, 2004

9. Coyle P, Philcox JC, Carey LC and Rofe AM: Metallothionein: The multipurpose protein. Cell Mol Life Sci 59: 627-647, 2002.

10. Maremanda KP, Khan S and Jena G: Zinc protects cyclophosphamide-induced testicular damage in rat: Involvement of metallothionein, tesmin and Nrf2. Biochem Biophys Res Commun 445: 591-596, 2014.

11. Oh JH, Yang JO, Hahn Y, Kim MR, Byun SS, Jeon YJ, Kim JM, Song KS, Noh SM, Kim S, et al: Transcriptome analysis of human gastric cancer. Mamm Genome 16: 942-954, 2005.

12. Fic M, Pula B, Rogala K and Dziegiel P: Role of metallothionein expression in gastrointestinal cancers. Postepy Biol Komorki 40: 5-20, 2013.

13. Grzegrzolka J, Gomulkiewicz A, Olbromski M, Glatzel-Plucinska N, Piotrowska A, Ratajczak-Wielgomas K, Rzechonek A, Podhorska-Okolow M, Krawczuk Z and Dziegiel P: Expression of tesmin (MTL5) in non small cell lung cancer: A preliminary study. Oncol Rep 42: 253-262, 2019.

14. Jankowska-Konsur A, Kobierzycki C, Reich A, Grzegrzolka J, Maj J and Dziegiel P: Expression of MCM-3 and MCM-7 in primary cutaneous T-cell lymphomas. Anticancer Res 35: 6017-6026, 2015.

15. Yu S, Wang G, Shi Y, Xu H, Zheng Y and Chen Y: MCMs in Cancer: Prognostic Potential and Mechanisms. Anal Cell Pathol (Amst) 2020: 3750294, 2020.

16. Honeycutt KA, Chen Z, Koster MI, Miers M, Nuchtern J, Hicks J, Roop DR and Shohet JM: Deregulated minichromosomal maintenance protein MCM7 contributes to oncogene driven tumorigenesis. Oncogene 25: 4027-4032, 2006.

17. Mukherjee G,Muralidhar B,Bafna UD,Laskey RA and Coleman N: MCM immunocytochemistry as a first line cervical screening test in developing countries: A prospective cohort study in a regional cancer centre in India. Br J Cancer 96: 1107-1111, 2007. 
18. Liu YZ, Wang BS, Jiang YY, Cao J, Hao JJ, Zhang Y, Xu X, Cai Y and Wang MR: MCMs expression in lung cancer: Implication of prognostic significance. J Cancer 8: 3641-3647, 2017.

19. Wang H, Zhou C, Su B, Teng G, Zheng Y, Zhou X, Guo L, $\mathrm{Xu} F$ and Wang X: MCM7 expression is correlated with histological subtypes of lung adenocarcinoma and predictive of poor prognosis. Int J Clin Exp Pathol 10: 11747-11753, 2017.

20. Kobierzycki C, Pula B, Wojnar A, Podhorska-Okolow M and Dziegiel P: Tissue microarray technique in evaluation of proliferative activity in invasive ductal breast cancer. Anticancer Res 32: 773-777, 2012.

21. Remmele W and Stegner HE: Recommendation for uniform definition of an immunoreactive score (IRS) for immunohistochemical estrogen receptor detection (ER-ICA) in breast cancer tissue. Pathologe 8: 138-140, 1987 (In German).

22. Livak KJ and Schmittgen TD: Analysis of relative gene expression data using real-time quantitative PCR and the 2(- $\Delta \Delta \mathrm{C}(\mathrm{T}))$ method. Methods 25: 402-408, 2001.

23. NowinskaK,JablonskaK,Pawelczyk K,Piotrowska A,Partynska A, Gomulkiewicz A, Ciesielska U, Katnik E, Grzegrzolka J, Glatzel-Plucinska N, et al: Expression of Irisin/FNDC5 in Cancer Cells and Stromal Fibroblasts of Non-small Cell Lung Cancer. Cancers (Basel) 11: 11, 2019.

24. Olbromski M, Rzechonek A, Grzegrzolka J, Glatzel-Plucinska N Chachaj A, Werynska B, Podhorska-Okolow M and Dziegiel P: Influence of miR-7a and miR-24-3p on the SOX18 transcript in lung adenocarcinoma. Oncol Rep 39: 201-208, 2018.
25. Olbromski M, Grzegrzolka J, Jankowska-Konsur A, Witkiewicz W, Podhorska-Okolow $M$ and Dziegiel P: MicroRNAs modulate the expression of the SOX18 transcript in lung squamous cell carcinoma. Oncol Rep 36: 2884-2892, 2016.

26. Vidarsdottir H, Tran L, Nodin B, Jirström K, Planck M, Jönsson P, Mattsson JSM, Botling J, Micke P and Brunnström H: Immunohistochemical profiles in primary lung cancers and epithelial pulmonary metastases. Hum Pathol 84: 221-230, 2019.

27. Sanada H, Seki N, Mizuno K, Misono S, Uchida A, Yamada Y, Moriya S, Kikkawa N, Machida K, Kumamoto T, et al: Involvement of dual strands of miR-143 (miR-143-5p and miR-143-3p) and their target oncogenes in the molecular pathogenesis of lung adenocarcinoma. Int J Mol Sci 20: 4482, 2019.

28. Fuller PJ: Novel interactions of the mineralocorticoid receptor. Mol Cell Endocrinol 408: 33-37, 2015.

29. Rogerson FM, Yao YZ, Young MJ and Fuller PJ: Identification and characterization of a ligand-selective mineralocorticoid receptor coactivator. FASEB J 28: 4200-4210, 2014.

(i) (5) This work is licensed under a Creative Commons Attribution-NonCommercial-NoDerivatives 4.0 International (CC BY-NC-ND 4.0) License. 